\title{
SETDB1 mediated FosB expression increases the cell proliferation rate during anticancer drug therapy
}

\author{
Han-Heom $\mathrm{Na}^{1}$, Hee-Jung Noh ${ }^{1}$, Hyang-Min Cheong ${ }^{2}$, Yoonsung Kang ${ }^{3}$ E Keun-Cheol Kim ${ }^{1, *}$ \\ ${ }^{1}$ Department of Biological Sciences, College of Natural Sciences, Kangwon National University, Chuncheon 24341, ${ }^{2}$ Division of Respiratory \\ Viruses, Center for Disease Control and Prevention, Korea National Institute of Health, ${ }^{3}$ Institute for Diagnostic Markers, Eudipia Inc, \\ Osong 28160, Korea
}

\begin{abstract}
The efficacy of anticancer drugs depends on a variety of signaling pathways, which can be positively or negatively regulated. In this study, we show that SETDB1 HMTase is down-regulated at the transcriptional level by several anticancer drugs, due to its inherent instability. Using RNA sequence analysis, we identified FosB as being regulated by SETDB1 during anticancer drug therapy. Fos $B$ expression was increased by treatment with doxorubicin, taxol and siSETDB1. Moreover, FosB was associated with an increased rate of proliferation. Combinatory transfection of siFosB and siSETDB1 was slightly increased compared to transfection of siFosB. Furthermore, FosB was regulated by multiple kinase pathways. ChIP analysis showed that SETDB1 and H3K9me3 interact with a specific region of the FosB promoter. These results suggest that SETDB1mediated FosB expression is a common molecular phenomenon, and might be a novel pathway responsible for the increase in cell proliferation that frequently occurs during anticancer drug therapy. [BMB Reports 2016; 49(4): 238-243]
\end{abstract}

\section{INTRODUCTION}

Treatment with anticancer drugs maximizes cell death by regulating various signaling pathways $(1,2)$. The apoptosis-related genes $\mathrm{p} 53$ and $\mathrm{Bcl} 2$ can be regulated by anticancer drug treatment, including taxol or doxorubicin $(3,4)$. Anticancer drugs can also activate the phosphoinositol-3-kinase (PI3K)/Akt and mitogen-activated protein kinase signaling pathways to induce cell proliferation or resistance, indicating that the drugs involve a variety of altered gene expressions related with both positive or negative effects $(5,6)$.

*Corresponding author. Tel: +82-33-250-8532; Fax: +82-33-2595665; E-mail: kckim@kangwon.ac.kr

http://dx.doi.org/10.5483/BMBRep.2016.49.4.031

Received 15 February 2016, Revised 22 February 2016, Accepted 1 March 2016

Keywords: Anticancer drugs, Doxorubicin, Drug resistance, FosB, SETDB1
The first step of gene expression is genome accessing of transcription machinery in the three-dimensional chromatin structure $(7,8)$. HMTases have been described in regulating a variety of cellular processes including development, differentiation, and pathogenesis $(9,10)$. SUV39H1, a mammalian HMTase of $\mathrm{H} 3 \mathrm{~K} 9 \mathrm{me} 3$ (histone $\mathrm{H} 3$ at lysine 9 tri-methylation), is recruited to double-strand breaks to transiently increase the levels of H3K9me3 in open chromatin domains, suggesting that SUV39H1 plays a critical role in stabilizing repressive chromatin to create an efficient template for the DNA repair machinery (11). EZH2, a HMTase for H3K27me3, is important for developmental regulators to maintain lineage integrity and retinal progenitor proliferation, as well as regulating the timing of late differentiation (12). SETDB1 is required for myogenic differentiation as well as transcriptional silencing by SETDB1, in embryonic stem cells $(13,14)$.

SETDB1 is the mammalian HMTase that methylates H3K9 and participates in heterochromatin compaction and gene repression during cellular processes $(15,16)$. Our previous studies have suggested that SETDB1 is negatively regulated during anticancer drug treatment using 3-deazaneplanocin A (DZNep) or taxol $(17,18)$. This down-regulation was controlled at the transcriptional level. In the case of taxol treatment, SETDB1 gene expression is regulated by co-occupancy of p53 and $\mathrm{SUV} 39 \mathrm{H} 1$ at the promoter region. SETDB1 is recurrently amplified in melanoma, and accelerates melanoma formation in a zebrafish model, implicating SETDB1 as an oncogenic chromatin factor in regulating tumorigenesis (19). Ectopic expression of SETDB1 in A549 cells significantly promoted cell invasion, strongly suggesting that SETDB1 is an oncogene (20). The collective data suggest that SETDB1 is an important HMTase that catalyzes a change in chromatin structure and gene expression during tumorigenesis. SETDB1 regulates downstream target genes to maximize its cellular responses. For example, the HOX gene is transcriptionally dysregulated in response to increased levels of SETDB1 (19). Mouse setdb1 protein suppresses interleukin- 2 production in mouse immune cells (21). Repressive complex containing setdb1 directly binds with the promoter of target genes, and methylates H3K9me3 (22). However, the functional importance of SETDB1 on the regulation of gene expression during anticancer drug treatment

ISSN: 1976-670X (electronic edition)

Copyright (c) 2016 by the The Korean Society for Biochemistry and Molecular Biology

(c) This is an open-access article distributed under the terms of the Creative Commons Attribution Non-Commercial License (http://creativecommons.org/licenses/by-nc/4.0) which permits unrestricted non-commercial use, distribution, and reproduction in any medium, provided the original work is properly cited. 
remains unclear.

Here, we provide evidence that many anticancer drugs commonly regulate the SETDB1 HMTase during therapy, and that the SETDB1 protein has inherently unstable characteristics. We also suggest that the FosB oncogene, as a SETDB1 mediated target gene, might have an important functional meaning via SETDB1 HMTase during anticancer drug therapy.

\section{RESULTS}

\section{Various anticancer drugs target SETDB1 down-regulation}

To examine whether SETDB1 is a common target HMTase for different anticancer drugs, A549 cells were treated with taxol
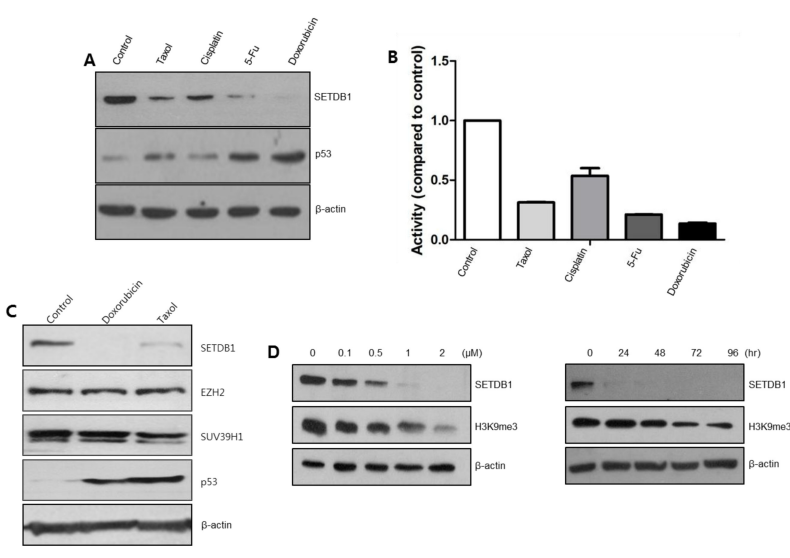

Fig. 1. SETDB1 is commonly regulated by various anticancer drugs. (A) A549 cells were treated with various anticancer drugs including taxol $(0.5 \mu \mathrm{M})$, cisplatin $(2 \mu \mathrm{M}), 5-\mathrm{FU}(5 \mu \mathrm{M})$, and doxorubicin $(2$ $\mu \mathrm{M})$. SETDB1 protein was down-regulated, whereas p53 protein was up-regulated in these treatments. (B) Luciferase assay was performed following anticancer drug treatment after pGL3-SETDB1-p-Luc transfection. (C) Western blots were done following doxorubicin or taxol treatment. SETDB1 was decreased, p53 was increased, but EZH2 or SUV39H1 displayed no change in protein levels. (D) Doxorubicin was treated in a dose- or time-dependent manner in A549 cells. H3K9me3 levels decreased, consistent with SETDB1 down-regulation.
$(0.5 \mu \mathrm{M})$, cisplatin $(2 \mu \mathrm{M})$, 5-fluorouracil (5-FU; $5 \mu \mathrm{M})$, and doxorubicin $(2 \mu \mathrm{M})$. SETDB1 protein was down-regulated by most anticancer drugs, and these drugs induced p53 protein production (Fig. 1A). Luciferase assay results showed that most anticancer drugs down-regulated the promoter activity of SETDB1 compared to control, indicating that SETDB1 gene expression was directly regulated at the transcription level (Fig. 1B). Next, we examined whether other HMTases could also be regulated by doxorubicin. EZH2 or SUV39H1 were not regulated, suggesting that anticancer drugs target only SETDB1 HMTase (Fig. 1C). SETDB1 down-regulation by doxorubicin also affected the histone methylation status (Fig. 1D). These results implied that anticancer drug-mediated SETDB1 down-regulation is the basis for the induction of cell death.

\section{SETDB1 is a relatively unstable HMTase}

To examine the stability of the SETDB1 protein, we treated anticancer drugs in combination with the protein synthesis inhibitor cycloheximide (CHX). SETDB1 was slightly decreased in the presence of $\mathrm{CHX}$ and severely decreased by the combination treatment of anticancer drugs and CHX (Fig. 2A). P53 protein was also decreased by $\mathrm{CHX}$ treatment. Even though anticancer drug treatment induced the $\mathrm{p} 53$ protein, it was decreased by the anticancer drugs in combination with $\mathrm{CHX}$. However, the EZH2 or $\mathrm{SUV} 39 \mathrm{H} 1$ remained unchanged by the $\mathrm{CHX}$ treatment. Interestingly, the RNA synthesis inhibitor actinomycin D, down-regulated the SETDB1 protein level, increased p53 protein level, but had no effect on EZH2 or SUV39H1 (Fig. 2B). This data indicates that SETDB1 is a highly unstable protein, and could thus be a target protein for anticancer drugs.

\section{FosB as a common target gene for anticancer drugs and SETDB1}

Our intent was to identify common target genes for SETDB1 and anticancer drugs. Total RNA was prepared from three experimental groups: doxorubicin-treated, taxol-treated, and siSETDB1-transfected A549 cells. Down-regulation of SETDB1 expression was confirmed by siSETDB1 transfection (Fig. 3A). RNA sequence analysis shows that 1576 genes $(P<0.05)$
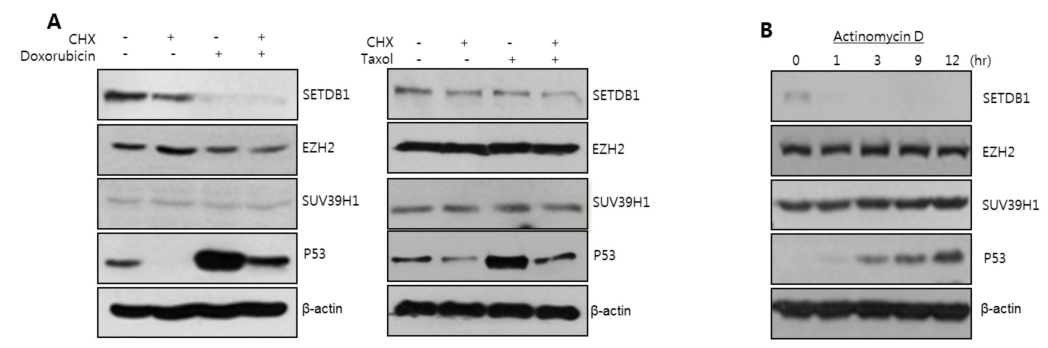

Fig. 2. SETDB1 is a relatively unstable protein. (A) Cycloheximide (CHX), a protein synthesis inhibitor, was pretreated for 12 hr prior to doxorubicin or taxol treatment. Anticancer drug was treated for $12 \mathrm{hr}$. SETDB1 down-regulation was aggravated in the combination treatment, whereas other HMTases were not. (B) SETDB1 protein was decreased in the treatment of the RNA synthesis inhibitor, actinomycin D. Other HMTases were not changed in the same treatment. 
were significantly increased in the doxorubicin-treated group, 428 genes $(\mathrm{P}<0.05)$ in the taxol-treated group, and 58 genes $(\mathrm{P}<0.05)$ in the siSETDB1-transfected group (Fig. 3B). Nine genes (FOSB, EGR2, JUN, DACT3, ABCD2, ATP1B2, AVPR1A, GFOD1, and ZFPM2) were identified as common target genes for doxorubicin, taxol, and SETDB1 (Fig. 3C). To confirm this, we performed RT-PCR analysis for FosB and EGR2. FosB and EGR2 were increased in anticancer drug treatments and siSETDB1-transfection (Fig. 3D). Western blot analysis using FosB antibody revealed a dose-dependent increase in the FosB protein by doxorubicin treatment (Fig. 3E). FosB expression was increased up to $36 \mathrm{hr}$, and then decreased at $48 \mathrm{hr}$ in a time-dependent manner for doxorubicin. FosB protein also increased with taxol or etoposide treatment (Fig. 3F).

\section{Molecular connection for combinatory regulation between SETDB1and FosB}

To assess how FosB expression affected the proliferation of A549 cells, we performed the MTT assay after FosB transfection. Enforced FosB expression induced the increased proliferation rate of A549 cells, and the proliferation rate was maintained in the presence of doxorubicin (Fig. 4A). We also performed a MTT assay using siRNAs. Transfection of siFosB decreased the cell proliferation rate compared to siscramble transfection. However, combinatory transfection of siFosB and siSETDB1 was slightly increased compared to siFosB transfection (Fig. 4B). To analyze which kinase pathway regulated the FosB expression, we performed Western blot after kinase inhibitors treatment with doxorubicin. Most kinase inhibitors affected FosB expression, indicating FosB gene regulation by multiple signaling pathways (Fig. 4C). To further examine SETDB1 mediated FosB expression, we performed ChIP analysis (Fig. 4D). SETDB1 bound a specific region $(-267$ to -115 upstream) of the FosB promoter, whereas no binding occurred at the other two regions. $\mathrm{H} 3 \mathrm{~K} 9 \mathrm{me} 3$ binding affinity was also consistent with SETDB1 occupancy in this region. However, SETDB1 protein binding did not occur with doxorubicin treatment, implying that SETDB1 directly regulates FosB expression during anticancer drug treatment.

\section{DISCUSSION}

In this study, we focused on SETDB1 as a common target HMTase for various anticancer drugs, and on the putative SETDB1-FosB pathway during chemotherapy. We believe that SETDB1 acts as a common HMTase for anticancer drugs, because SETDB1 exhibits greater inherently instability as compared to other HMTases.

Another interesting aim was to identify the target genes, to examine the role of SETDB1 during anticancer drug therapy. This approach is important to clarify why many anticancer drugs target the SETDB1 HMTase. We identified nine candidate genes (FOSB, EGR2, JUN, DACT3, ABCD2, ATP1B2, AVPR1A, GFOD1, and ZFPM2). Among them, we confirmed that FosB expression was increased by anticancer drugs and SETDB1 HMTase. FosB (FB) murine osteosarcoma viral oncogene homolog B), a Fos gene family, encodes leucine zipper proteins that can dimerize with proteins of the JUN family to form the transcription factor complex AP-1 (23). FosB is activated through PI3K-Akt/protein kinase $\mathrm{B}$ pathway during nitric
A
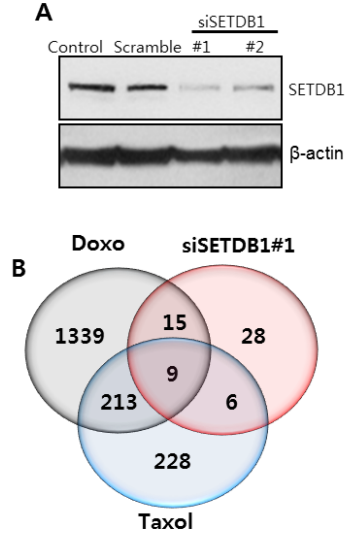

D

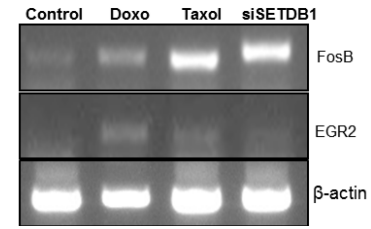

C

\begin{tabular}{|l|r|r|r|}
\hline \multirow{2}{*}{ gene (locus) } & \multicolumn{3}{c|}{ Fold induction } \\
\cline { 2 - 5 } & \multicolumn{1}{|c|}{ doxorubicin } & \multicolumn{1}{c|}{ taxol } & \multicolumn{1}{c|}{ siSETDB1 } \\
\hline FOSB (chr19:45971252-45978437) & 38.35296074 & 17.65680692 & 85.73417046 \\
\hline EGR2 (chr10:64571755-64578927) & 52.7915749 & 10.7446014 & 7.652844232 \\
\hline JUN (chr1:59246462-59249785) & 5.156927309 & 6.293373833 & 7.422057352 \\
\hline DACT3 (chr19:47150868-47220384) & 146.4073 & 5.962428 & 5.68227 \\
\hline ABCD2 (chr12:39945021-40013843) & 7.280214 & 8.936309 & 7.30407 \\
\hline ATP1B2 (chr17:7554253-7561089) & 42.81003 & 5.689885 & 6.0876 \\
\hline AVPR1A (chr12:63536538-63546590) & 24.10465 & 5.685146 & 6.69485 \\
\hline GFOD1 (chr6:13363586-13487869) & 31.73076 & 15.93559 & 5.33808 \\
\hline ZFPM2 (chr8:106331146-106816767) & 10.01319 & 8.937911 & 6.08704 \\
\hline
\end{tabular}

E

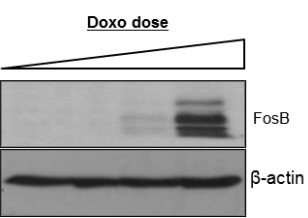

$\mathbf{F}$

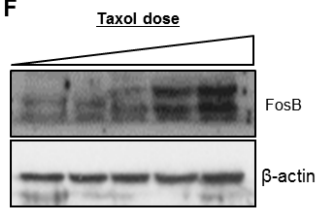

Doxo time

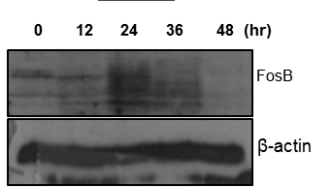

Etoposide dose

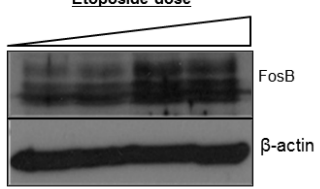

Fig. 3. Nine genes were identified as commonly regulated genes through RNA sequence analysis. (A) Two siSETDB1 were prepared and transfected into A549 cells. SETDB1 protein level was downregulated by siSETDB1 transfection. (B) Up-regulated genes were analyzed after doxorubicin, taxol, and siSETDB1 treatment. (C) Nine genes were identified as common target genes for doxorubicin, taxol, and SETDB1. Among them, FosB gene expression showed the highest change. (D) RT-PCR analysis was performed to check the expression of FosB and EGR2. (E) Western blot showed that doxorubicin treatment increased the FosB gene expression in a dose- or time-dependent manner. $(F)$ Treatment of taxol or etoposide increased the FosB gene expression in a dose-dependent manner. 
A

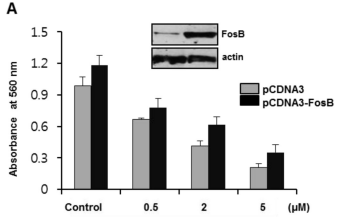

c

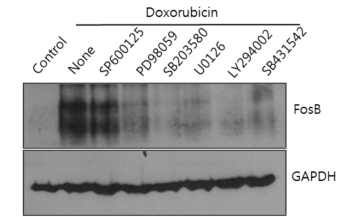

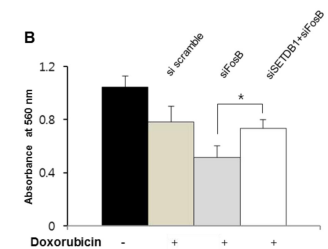

D Input IgG SETDB1 HзK9me3

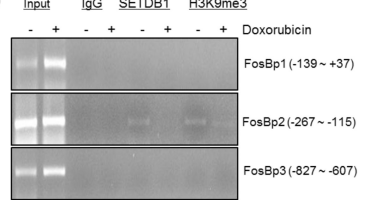

Fig. 4. SETDB1 mediated FosB expression changes the cell proliferation rate. (A) A549 cells were transfected with pCDNA3-FosB plasmid in $60 \mathrm{~mm}$ diameter dishes, after which they were seeded at a density of 2,000 cells/well in 96-well plates. The MTT assay was performed after doxorubicin treatment. (B) A549 cells were transfected with siRNA, and were treated with doxorubicin after seeding in 96-well plates for the MTT assay. Cell proliferation rate decreased with siFosB, but increased marginally in the combinatory transfection of siSETDB1and siFosB. $\left({ }^{* P}<0.05\right)$ (C) A549 cells were treated with various kinase inhibitors, along with doxorubicin. Western blot showed that most kinase inhibitors regulated the increased FosB gene expression by doxorubicin. (D) ChIP assay was performed with three primer sets for FosB promoter region, in the absence or presence of doxorubicin treatment. SETDB1 binding was detected in the specific regions $(-267$ to -115$)$. H3K9me3 occupancy was consistent in these regions.

oxide production in human neutrophils (24). The FosB/Jun activator protein-1 (AP-1) family is transcriptionally activated following exposure to environmental chemical and biological agents, in a mouse lung model (25). FosB has been found as alternative spliced transcript variants or multiple truncated forms (26). Even though the exact function of FosB remains unknown, full-length FosB may prevent or counteract the transcriptional effects of $\Delta F o s B$, perhaps through the modulation of other genes (27). Induction of FosB is likely one of the mechanisms underlying addiction (28). Increasing evidence indicates that this induction represents a positive homeostatic adaptation to chronic stress, since over-expression of $\triangle \mathrm{FosB}$ in this brain region promotes resilience to stress, whereas blockade of its activity promotes stress susceptibility (29). Anticancer drug treatment may lead to the down-regulation of acetylpolyamine oxidase (paox), which parallels an increase in the expression of the AP-1 factors, c-Jun and FosB (30). This FosB/Jun AP-1 family activation regulates $\Delta N p 73$-mediated resistance to chemotherapeutic drugs. Therefore, the FosB gene might be regulated by various environmental signals, at least partially, is associated with resistance or addiction on environ signals.

Suppression by siSETDB1 transfection reduced the cell proliferation rate, migration rate, and colony forming activity of glioma cells (31). Our data suggests that overexpression or suppression of FosB is clearly correlated with the cell proliferation rate. However, it is interesting to note that the status

of siSETDB1 and siFosB restored the cell proliferation rate, compared to siFosB transfection only. These results imply that there might be a combinatory effect of SETDB1 and FosB. The results of treatment with most kinase inhibitors suggest that certain pathway(s) modulate expression of SETDB1 and FosB, and therefore also the efficacy of anticancer drugs.

SETDB1 HMTase specifically bound to a defined region $(-267$ to -115$)$ of FosB genomic DNA. This region of the FosB promoter can be occupied by $\mathrm{CBX7}$, which is a key protein in carcinogenesis (32). CBX7, a polycomb protein member of the polycomb repressive complex 1 (PRC1), is downregulated in malignant grade and neoplastic stage in thyroid carcinomas; it also maintains important developmental genes in a transcriptionally repressed state (33). Therefore, we postulate that this region of FosB promoter might be a critical regulatory region for cellular proliferation rates.

There are many reports on FosB expression in various stimuli. However, this paper is the first to report the reverse expression between SETDB1 and FosB during anticancer drug treatment. Considering that FosB protein is regulated in drug addiction or is chronically produced by exposure to various stimuli, the FosB gene might be expressed for negative regulation during anticancer drug treatment. Collectively, the data demonstrate that SETDB1-FosB expression is a cue for a novel transcriptional mechanism of drug resistance or cell proliferation. Further studies will seek the functional mechanism on SETDB1-FosB pathway during therapy using anticancer drugs.

\section{MATERIALS AND METHODS}

\section{Cell cultures, antibodies, and reagents}

A549 human lung cancer cell line was purchased from the Korean Cell Bank. Cells were cultured in RPMI 1640 medium containing $10 \%$ fetal bovine serum, and maintained in a humidified incubator at $37^{\circ} \mathrm{C} / 5 \% \mathrm{CO}_{2}$. The anticancer drugs, cycloheximide $(\mathrm{CHX})$, actinomycin $\mathrm{D}$, and kinase inhibitors were obtained from Sigma Aldrich (Seoul, Korea). The drugs were dissolved in appropriate solvents according to the manufacturer's protocol. Antibodies for this experiment were purchased from the following companies: SETDB1 (Abcam, MA, USA), SUV39H1 (Upstate Biotechnology, NY, USA), EZH2 (Abcam, MA, USA), p53 (Santa Cruz Biotechnology, CA, USA), FosB (Santa Cruz Biotechnology, CA, USA), H3K9me3 (Abcam, MA, USA), and $\beta$-actin (Sigma-Aldrich).

\section{Westem blot}

Cells were lysed with RIPA buffer supplemented with Complete Mini protease inhibitor cocktail tablets (Roche, Seoul, Korea). The lysates were resolved on acrylamide gels and were transferred to a polyvinylidene fluoride membrane (Millipore, Seoul, Korea). Membranes were blocked with 5\% skim milk for $1 \mathrm{hr}$ at room temperature. After washing, the membranes were incubated with appropriate primary antibodies. The membranes were washed and then incubated 
with the appropriate secondary antibodies for $1 \mathrm{hr}$. The proteins were detected using a protein detection kit (Animal Genetic, Seoul, Korea).

\section{Luciferase assay}

The SETDB1 promoter construct was transfected into A549 cells using $5 \mu \mathrm{l}$ lipofectamine 2000 (Invitrogen, CA, USA). The cells were also co-transfected with the $\beta$-gal plasmid to normalize for transfection efficiency. Cells were treated with anticancer drugs after $12 \mathrm{hr}$ post-transfection, and harvested $12 \mathrm{hr}$ after anticancer drugs treatment. Total cell lysates were prepared using 1X Passive Lysis Buffer (Promega, MI, USA), and were assessed for firefly luciferase activity. Luminescence was monitored using a Glomax 96 microplate luminometer (Promega, MI, USA).

\section{RNA extraction, RNA-Sequencing, and RT-PCR}

Small interfering (si)RNA for SETDB1 or FosB were designed and purchased from Bioneer Inc. (Daejeon, Korea). siRNA was transfected into A549 cells using lipofectamine 2000. After $48 \mathrm{hr}$ post-transfection, total RNA was isolated using a TRIZOL kit (Duchefa, Haarlem, Netherlands). RNA sequence analysis was performed at BML Inc. (Korea). For RT-PCR experiment, complementary DNA (cDNA) was synthesized with hexamer from total RNA. Conventional PCR was performed as described in a previous study (12). cDNA was mixed with specific primer sets in $0.2 \mathrm{mM}$ dNTP, $1 \cup$ Taq polymerase, buffer containing $1.5 \mathrm{mM}$ $\mathrm{MgCl}_{2}$ (Enzynomics, Seoul, Korea). The primer sequences were SETDB1 S1 5'-TTA ACA CAG GCC CTG AAT TTC T-3' SETDB1 AS1 5'-TAC CCC TGT GGG TAG ACA CTC T-3', FosB S1 5'-TAC TCC ACA CCA GGC ATG AG-3, AS' 5'-CTT CGT AGG GGA TCT TGC AG-3', EGR2 S1 5'-ATT CTG AGG CCT CGC AAG TA-3', AS' 5'-TGC TTT TCC GCT CTT TCT GT-3', actin S1 5'-GTG GGG CGC CCC AGG CAC CAG GGC-3', and actin AS1 5'-CTC CTT AAT GTC ACG CAC GAT TTC-3'. PCR reactions were carried out in a Perkin Elmer Thermal Cycler 9600 (Applied Biosystems, MA, USA). PCR products were resolved in $1.5 \%$ agarose gels.

\section{Viability assay}

Growth inhibitory effect was measured using a MTT (dimethyl thiozolyl-2',5'-diphenyl-2-H-tetrazolium bromide) assay (34). MTT purchased from Sigma-Aldrich was dissolved in phosphate-buffered saline (PBS). The FosB plasmid was provided by Korea Human Gene Bank, and subcloned into pCDNA3 expression vector. After cells were transfected with pCDNA3-FosB plasmid in $60 \mathrm{~mm}$ dishes, they were seeded at 2,000 cells/well in 96-well plates. The next day, cells were treated with various concentrations of doxorubicin, and were incubated in MTT solution for $2 \mathrm{hr}$ at $37^{\circ} \mathrm{C}$. The MTT solution was replaced with $100 \mu \mathrm{l}$ of dimethylsulfoxide to dissolve the formazan crystals. Absorbance was measured using a microplate reader (Bio-Rad, CA, USA) at a wavelength of $570 \mathrm{~nm}$. This experiment was conducted in triplicate.

\section{ChIP assay}

A previously described protocol was used (24). Briefly, cells were cross-linked with $1 \%$ formaldehyde and sonicated in lysis buffer. The lysate was incubated with the appropriate antibody overnight, and washed sequentially with a low salt buffer (0.1\% SDS, 1\% Triton X-100, 2 mM EDTA, 20 mM Tris-HCl, $150 \mathrm{mM} \mathrm{NaCl})$, high salt buffer $(0.1 \%$ SDS, $1 \%$ Triton X-100, $2 \mathrm{mM}$ EDTA, $20 \mathrm{mM}$ Tris- $\mathrm{HCl}, 500 \mathrm{mM} \mathrm{NaCl}$ ), lithium chloride buffer $(0.25 \mathrm{M} \mathrm{LiCl}, 1 \% \mathrm{NP}-40,1 \mathrm{mM}$ EDTA, $10 \mathrm{mM}$ Tris- $\mathrm{HCl}$ ). The beads were eluted with $1 \%$ SDS and $0.1 \mathrm{M}$ $\mathrm{NaHCO}_{3}$ at $65^{\circ} \mathrm{C}$. A DNA extract was then used for PCR amplification. The primers used for PCR amplification of FosB promoter were FosBp1-S1; 5'- GCT GAG AAC GGA AAA CAA TC -3', FosBp1-AS1; 5' - TGT GGA CAT TAC CTG AAG CC -3', FosBp2-S1; 5'-GCT GAG AAC GGA AAA CAA TC-3', FosBp2-AS1; 5'-TGT GGA CAT TAC CTG AAG CC-3', FosBp3-S1; 5'-GCT GAG AAC GGA AAA CAA TC-3', and FosBp3-AS1; 5'- TGT GGA CAT TAC CTG AAG CC -3'.

\section{Statistical analysis}

Data are presented as means \pm SD. The data were evaluated statistically by single-factor analysis of variance (ANOVA) for multiple groups, or t-test for two groups. Statistically significant differences between experimental groups were considered at $\mathrm{P}<0.05$.

\section{ACKNOWLEDGEMENTS}

This work was supported by the LINC R\&BD program (C1012278-01-01) and a fund (C1012325-01-01) from AURI (Korea association of University, Research Institute and Industry). This work was also supported by Basic Science Research Program through the National Research Foundation of Korea (NRF-2010-0022705), Korea

\section{REFERENCES}

1. Giussani $P$, Tringali $C$, Riboni L, Viani $P$ and Venerando $B$ (2014) Sphingolipids: key regulators of apoptosis and pivotal players in cancer drug resistance. Int J Mol Sci 15, 4356-4392

2. Yang W, Park IJ, Yun H et al (2014) AMP-activated protein kinase alpha2 and E2F1 transcription factor mediate doxorubicin-induced cytotoxicity by forming a positive signal loop in mouse embryonic fibroblasts and non-carcinoma cells. J Biol Chem 289, 4839-4852

3. Korbakis D and Scorilas A (2012) Quantitative expression analysis of the apoptosis-related genes BCL2, BAX and BCL2L12 in gastric adenocarcinoma cells following treatment with the anticancer drugs cisplatin, etoposide and taxol. Tumour Biol 33, 865-875

4. Choi YH and Yoo YH (2012) Taxol-induced growth arrest and apoptosis is associated with the upregulation of the Cdk inhibitor, p21WAF1/CIP1, in human breast cancer 
cells. Oncol Rep 28, 2163-2169

5. Liu Z, Zhu G, Getzenberg RH and Veltri RW (2015) The Upregulation of PI3K/Akt and MAP Kinase Pathways is Associated with Resistance of Microtubule-Targeting Drugs in Prostate Cancer. J Cell Biochem 116, 1341-1349

6. Jung KA, Choi BH and Kwak MK (2015) The c-MET/PI3K signaling is associated with cancer resistance to doxorubicin and photodynamic therapy by elevating BCRP/ ABCG2 expression. Mol Pharmacol 87, 465-476

7. Guetg C and Santoro R (2012) Formation of nuclear heterochromatin: the nucleolar point of view. Epigenetics 7, 811-814

8. Meier K and Brehm A (2014) Chromatin regulation: how complex does it get? Epigenetics 9, 1485-1495

9. Kamiunten T, Ideno H, Shimada A et al (2015) Coordinated expression of $\mathrm{H} 3 \mathrm{~K} 9$ histone methyltransferases during tooth development in mice. Histochem Cell Biol 143, 259-266

10. Zhang $Y$ and Tong $T$ (2014) FOXA1 antagonizes EZH2mediated CDKN2A repression in carcinogenesis. Biochem Biophys Res Commun 453, 172-178

11. Ayrapetov MK, Gursoy-Yuzugullu O, Xu C, Xu Y and Price BD (2014) DNA double-strand breaks promote methylation of histone $\mathrm{H} 3$ on lysine 9 and transient formation of repressive chromatin. Proc Natl Acad Sci U S A $111,9169-9174$

12. Zhang J, Taylor RJ, La Torre A et al (2015) Ezh2 maintains retinal progenitor proliferation, transcriptional integrity, and the timing of late differentiation. Dev Biol 403, 128-138

13. Song YJ, Choi JH and Lee $\mathrm{H}$ (2015) Setdb1 is required for myogenic differentiation of $\mathrm{C} 2 \mathrm{C} 12$ myoblast cells via maintenance of MyoD expression. Mol Cells 38, 362-372

14. Thompson PJ, Dulberg V, Moon KM et al (2015) hnRNP K coordinates transcriptional silencing by SETDB1 in embryonic stem cells. PLoS Genet 11, e1004933

15. Schultz DC, Ayyanathan K, Negorev D, Maul GG and Rauscher FJ (2002) SETDB1: a novel KAP-1-associated histone $\mathrm{H} 3$, lysine 9-specific methyltransferase that contributes to HP1-mediated silencing of euchromatic genes by KRAB zinc-finger proteins. Genes Dev 16, 919-932

16. Wu PC, Lu JW, Yang JY et al (2014) H3K9 histone methyltransferase, KMT1E/SETDB1, cooperates with the SMAD2/3 pathway to suppress lung cancer metastasis. Cancer Res 74, 7333-7343

17. Lee JK and Kim KC (2013) DZNep, inhibitor of S-adenosylhomocysteine hydrolase, down-regulates expression of SETDB1 H3K9me3 HMTase in human lung cancer cells. Biochem Biophys Res Commun 438, 647-652

18. Noh HJ, Kim KA and Kim KC (2014) p53 down-regulates SETDB1 gene expression during paclitaxel induced-cell death. Biochem Biophys Res Commun 446, 43-48

19. Ceol CJ, Houvras Y, Jane-Valbuena J et al (2011) The histone methyltransferase SETDB1 is recurrently amplified in melanoma and accelerates its onset. Nature 471, 513-517

20. Rodriguez-Paredes M, Martinez de Paz A, Simo-Riudalbas L et al (2014) Gene amplification of the histone methyltransferase SETDB1 contributes to human lung tumorigenesis. Oncogene 33, 2807-2813
21. Wakabayashi Y, Tamiya T, Takada I et al (2011) Histone 3 lysine 9 (H3K9) methyltransferase recruitment to the interleukin-2 (IL-2) promoter is a mechanism of suppression of IL-2 transcription by the transforming growth factor-beta-Smad pathway. J Biol Chem 286, 35456-35465

22. Martin FJ, Xu Y, Lohmann F et al (2015) KMT1E-mediated chromatin modifications at the FcgammaRllb promoter regulate thymocyte development. Genes Immun 16, 162-169

23. Ramachandran A, Gong EM, Pelton K et al (2011) FosB regulates stretch-induced expression of extracellular matrix proteins in smooth muscle. Am J Pathol 179, 2977-2989

24. Ratajczak-Wrona W, Jablonska E, Garley M et al (2014) $\mathrm{PI} 3 \mathrm{~K}-\mathrm{Akt} / \mathrm{PKB}$ signaling pathway in neutrophils and mononuclear cells exposed to $\mathrm{N}$-nitrosodimethylamine. J Immunotoxicol 11, 231-237

25. Win-Shwe TT and Fujimaki H (2015) Activation of transcription factors in a mouse lung following exposure to environmental chemical and biological agents. J Toxicol Sci 40, 559-568

26. Hong S, Skaist AM, Wheelan SJ and Friedman AD (2011) AP-1 protein induction during monopoiesis favors C/EBP: AP-1 heterodimers over C/EBP homodimerization and stimulates FosB transcription. J Leukoc Biol 90, 643-651

27. Lazenka MF, David BG, Lichtman AH, Nestler EJ, Selley DE and Sim-Selley LJ (2014) Delta FosB and AP-1-mediated transcription modulate cannabinoid $\mathrm{CB}(1)$ receptor signaling and desensitization in striatal and limbic brain regions. Biochem Pharmacol 91, 380-389

28. Ruffle JK (2014) Molecular neurobiology of addiction: what's all the (Delta)FosB about? Am J Drug Alcohol Abuse 40, 428-437

29. Vialou V, Thibault M, Kaska S et al (2015) Differential induction of FosB isoforms throughout the brain by fluoxetine and chronic stress. Neuropharmacology 99, 28-37

30. Bunjobpol W, Dulloo I, Igarashi K, Concin N, Matsuo K and Sabapathy K (2014) Suppression of acetylpolyamine oxidase by selected AP-1 members regulates DNp73 abundance: mechanistic insights for overcoming DNp73mediated resistance to chemotherapeutic drugs. Cell Death Differ 21, 1240-1249

31. Spyropoulou A, Gargalionis A, Dalagiorgou G et al (2014) Role of histone lysine methyltransferases SUV $39 \mathrm{H} 1$ and SETDB1 in gliomagenesis: modulation of cell proliferation, migration, and colony formation. Neuromolecular Med 16, 70-82

32. Pallante $P$, Sepe $R$, Federico A, Forzati $F$, Bianco $M$ and Fusco A (2014) CBX7 modulates the expression of genes critical for cancer progression. PLoS One 9, e98295

33. Scott CL, Gil J, Hernando E et al (2007) Role of the chromobox protein CBX7 in lymphomagenesis. Proc Nat Acad Sci U S A 104, 5389-5394

34. Im CN, and Seo JS (2014) Overexpression of tumor necrosis factor receptor-associated protein 1 (TRAP1), leads to mitochondrial aberrations in mouse fibroblast $\mathrm{NIH} / 3 \mathrm{~T} 3$ cells. BMB Rep 47, 280-285

35. Shang Y, Hu X, DiRenzo J, Lazar MA and Brown M (2000) Cofactor dynamics and sufficiency in estrogen receptor-regulated transcription. Cell 103, 843-852 